\title{
Advances and Frontiers in Metamaterials
}

\author{
Huanyang Chen* \\ Department of Physics and Institute of Electromagnetics and Acoustics, Xiamen University, Xiamen, China
}

Keywords: effective medium theory, metamaterials, Metasurfaces, plasmonics, transformation optics, Topological photonics or acoustics, Metamaterials with Artificial Intelligence

In optics, the direction of light propagation is manipulated by the shapes of objects and their refractive indexes, thus achieving optical devices such as convex and concave lenses. With increased understanding of materials, researchers are exploring deeper into the microscopic fields and have focused on microstructures of materials. In condensed matters, crystals are solid-state materials with constituents and atoms arranged periodically in space resulting in a variety of extraordinary properties. When it comes to the classical systems, structures of subwavelength scales also exhibit many novel phenomena, such as negative refraction, invisible cloaking and superresolution imaging, and they are usually analyzed in the fields of wave optics or acoustics.

In 1987, Eli Yablonovitch (Yablonovitch, 1987) and Sajeev John (John, 1987) first proposed the concept of photonic crystals to describe the propagation of optical waves in the refraction indexmodulated periodic structures, as well as the theory of photonic band structures, which paves a new way to manipulate light using photonic crystals and explore wave effects therein and applications (Cregan, 1999; Knight, 2003). Subsequently, a similar concept in acoustic systems was also presented in 1993, which is known as sonic/phononic crystals (Kushwaha et al., 1993; Sigalas and Economou, 1993) and has also been extended to practical applications (Miniaci et al., 2017).

To better manipulate the wave-propagating behaviors, functional microstructures were designed

\section{OPEN ACCESS}

Edited and reviewed by:

Nicola Maria Pugno,

University of Trento, Italy

*Correspondence:

Huanyang Chen

kenyon@xmu.edu.cn

Specialty section:

This article was submitted to Metamaterials,

a section of the journal

Frontiers in Materials

Received: 24 March 2021 Accepted: 22 April 2021 Published: 07 May 2021

Citation:

Chen H (2021) Advances and Frontiers in Metamaterials.

Front. Mater. 8:685025. doi: $10.3389 /$ fmats.2021.685025 to realize various extraordinary properties. These microstructures are known as metamaterials. Sir John Pendry proposed the idea of wire mesh (or cut-wire) systems to achieve negative values of effective permittivities in microwaves (Pendry et al., 1996) in 1996, and other periodically arranged local resonant structures (such as split resonant rings) for negative values of effective permeabilities in microwaves (Pendry et al., 1999) in 1999. Soon afterwards, metamaterials with simultaneously negative permittivity and permeability were realized and verified in microwave experiments by Smith et al. (Smith et al., 2000), as well as other negative refractive index materials (Smith, 2004). Other important local resonant structures in microwaves were also proposed, such as $\mathrm{H}$-fractal structures (Wen et al., 2002). In addition, for acoustic systems, metamaterials with subwavelength scales have developed rapidly using locally resonant sonic structures (Liu, 2000), which exhibits spectral phononic band gaps in the long wavelength range and could be regarded as effective densities and elastic moduli. These properties pave the way for various applications of metamaterials, including perfect lenses (Pendry, 2000). In 2006, Leonhardt and Sir John Pendry et al. initiated the field of transformation optics (Leonhardt, 2006; Pendry, 2006) based on the form invariance of Maxwell's equations under coordinate transformations. With the development of the above metamaterials with customized material parameters, invisibility cloaks (Schurig et al., 2006; Chen et al., 2007; Liu et al., 2009) and other intriguing devices (Chen et al., 2010) were implemented. Such a concept was later on extended to acoustics (Chen and Chan, 2007; Cummer and Schurig, 2007), surface water waves (Farhat et al., 2008; Chen et al., 2009; Li et al., 2018), and thermal dynamics (Fan et al., 2008; Schittny et al., 2013). Since then, the associated topics from metamaterials have greatly attracted research interests and have been studied for decades. 
Therefore, we have launched a new specialty section dedicated to Metamaterials in the open access journal Frontiers in Materials with the aim to bring the whole community together. The specialty section accepts submissions across the breadth of metamaterial science and engineering, including but not limited to electromagnetic and optical metamaterials (Pendry, 2006), acoustic metamaterials (Cummer et al., 2016; Ma and Sheng, 2016), hydrodynamic metamaterials (Park et al., 2019; Zou et al., 2019) and thermal metamaterials (Han et al., 2014; Xu et al., 2014).

However, there are challenges to consider. For example, in the optical range, metamaterials have a large absorption owing to loss of metal and are usually not easy to fabricate due to their threedimensional structures, which have limited their promotion and applications. To overcome the challenges, metasurfaces emerged. In 2011, Federico Capasso et al. proposed generalized Snell's Law (GSL), which shows that when there exists a phase gradient along the interface of two media, anomalous reflection/refraction would happen. By using V-shaped metallic antennas with different abrupt phases, they experimentally demonstrated GSL (Yu et al., 2011). In 2012, L. Zhou et al. experimentally realized the conversion of propagating waves to surface waves by utilizing $\mathrm{H}$-shaped phase gradient metasurfaces (Sun et al., 2012). Since then, metasurfaces become a fast-growing research area. As two-dimensional counterparts of metamaterials, metasurfaces are composed of subwavelength meta-atoms and own extraordinary abilities to manipulate the wavefronts of electromagnetic waves (EMs) in free space and waveguides (Xu et al., 2016). Owing to their planner structure, metasurfaces are easy to fabricate compared with metamaterials, thus leading to more practical applications, such as mathematical operations (Silva et al., 2014), metalens (Aieta et al., 2015), and so on. As one of the most important applications of metasurfaces, metalens is composed of low loss dielectric materials and of higher efficiency property when compared with metallic metasurfaces, yet with a larger thickness. To balance the efficiency and thickness, metagratings (Xu et al., 2015) composed of both metal and dielectric were proposed to manipulate the wave propagations with diffraction theory and the associated higher-order diffraction law (Fu et al., 2019) that are beyond the GSL. Moreover, to make the functionalities of metasurfaces tunable, T. Cui et al. proposed coding metasurfaces by loading biased diodes into the unit cells (Cui et al., 2014). Due to their powerful abilities in the control of waves, the concept of metasurfaces has also been extended to other dynamic systems, such as acoustic (Assouar et al., 2018) and elastic (Li et al., 2020) waves.

To make metamaterials thin, we get metasurfaces. In contrast, to make the scale lager, we get crystals. Interestingly, the photonic/sonic crystals and metamaterials can not only adjust their effective medium and phase characteristics by materials and structures, but also define their topological features. In recent years, topological materials have become research hotspots in condensed matter physics for their exotic propagation behavior of surface states, which have been extended to the study of topological photonics/acoustics and injected new vitality into the research of photonic and sonic/acoustic crystals. In 2008,
Haldane and Raghu observed the nontrivial band structure in a two-dimensional photonic crystal by breaking time-reversal symmetry, and verified the existence of robust chiral edge states (Haldane and Raghu, 2008). The topological invariants have also been defined in photonic systems, and bringing about many exotic topological effects, such as photonic topological insulators, semimetals and higher-order topological insulators (Hasan and Kane, 2010; Khanikaev et al., 2013; Ozawa et al., 2019; Kim et al., 2020; Liu et al., 2021). Various topological phases in acoustics and elasticity have also been demonstrated (Xiao et al., 2015; Yang et al., 2015; Yan et al., 2018; Ma et al., 2019) and are promoted to other classical systems.

Most recently, machine learning has been used to design and optimize metamaterials and photonic/acoustic crystals, which can achieve the desired structure parameters in highly efficient ways. Many theoretical and experimental works have demonstrated its applicability in photonics/acoustics, such as inversed designs (Molesky et al., 2018), ultrafast photonics (Genty et al., 2021), and metasurface cloaks (Qian et al., 2020).

Compared with the above artificial materials, the van der Waals (vdW) materials in nature have also been widely investigated, such as graphene, $\mathrm{MoO}_{3}$ and Transition metal dichalcogenides (TMD), resulting in the discovery of exotic phenomena. In recent years, twist electronics has become an emerging hot topic and attracted much attention, such as the anisotropic polaritons in vdW materials (Hu et al., 2020), magic angles and magic potentials in graphene and TMD (Cao et al., 2018; Tang et al., 2020; Park et al., 2021), which may display flat bands and realize topological superconductivity. We can see that these $\mathrm{vdW}$ materials can also present many interesting physical mechanisms due to their natural material properties, which is beyond the microstructures. Therefore, considering the intersections of novel materials and microstructures to give rise to new physics is a direction worth exploring, which may induce new discoveries in the near future.

Apart from the above manipulations for single physics field, realizing efficient control of elastic waves (Ge et al., 2018) and other multi-physics field (Ma et al., 2014) are very important for solving engineering problems, such as in 5G, sensing, energy and other fields. However, the designs of metamaterials are extremely complicated, in particular for elastic waves due to the coupling between transverse waves and longitudinal waves (Lai et al., 2011). In addition, the elastic wave equation does not have the form invariance under coordinate transformation (Milton et al., 2006), therefore it is not possible to propose a general transformation elastics (while there are indeed several reductions for simple designs (Brun et al., 2009; Farhat et al., 2009; Zhou and Chen, 2019). To compensate such weakness, an efficient way is to use Willis materials (Willis, 1981). And with the notion of metamaterials, they can be experimentally realized for elastic waves (Liu et al., 2019). Moreover, the elastic metamaterials can also be applied in seismic waves for earthquake systems (Brûlé et al., 2014; Yakovleva et al., 2021).

In conclusion, the fields of metamaterials and photonic/sonic crystals have developed rapidly during the past two decades to combine with new frontiers and spark new ideas, and have been applied to many novel optical devices. The field will continue to 
grow and surprise us in the future. In addition, not only is there interesting physics to be discovered by merging with natural materials, metamaterials will soon become a great part of the field of materials science and engineering. Therefore, the Metamaterials specialty section of Frontiers in Materials warmly welcomes submissions from physics, materials science, artificial intelligence, energy science, and engineering. The section will be an open access platform for the interdisciplinary community of metamaterials scientists and engineers.

\section{REFERENCES}

Aieta, F., Kats, M. A., Genevet, P., and Capasso, F. (2015). Multiwavelength Achromatic Metasurfaces by Dispersive Phase Compensation. Science 347, 1342-1345. doi:10.1126/science.aaa2494

Assouar, B., Liang, B., Wu, Y., Li, Y., Cheng, J.-C., and Jing, Y. (2018). Acoustic Metasurfaces. Nat. Rev. Mater. 3, 460-472. doi:10.1038/s41578-018-0061-4

Brûlé, S., Javelaud, E. H., Enoch, S., and Guenneau, S. (2014). Experiments on Seismic Metamaterials: Molding Surface Waves. Phys. Rev. Lett. 112, 133901. doi:10.1103/PhysRevLett.112.133901

Brun, M., Guenneau, S., and Movchan, A. B. (2009). Achieving Control of In-Plane Elastic Waves. Appl. Phys. Lett. 94, 061903. doi:10.1063/1.3068491

Cao, Y., Fatemi, V., Fang, S., Watanabe, K., Taniguchi, T., Kaxiras, E., et al. (2018). Unconventional Superconductivity in Magic-Angle Graphene Superlattices. Nature 556, 43-50. doi:10.1038/nature26160

Chen, H., and Chan, C. T. (2007). Acoustic Cloaking in Three Dimensions Using Acoustic Metamaterials. Appl. Phys. Lett. 91, 183518. doi:10.1063/1.2803315

Chen, H., Chan, C. T., and Sheng, P. (2010). Transformation Optics and Metamaterials. Nat. Mater. 9, 387-396. doi:10.1038/nmat2743

Chen, H., Wu, B.-I., Zhang, B., and Kong, J. A. (2007). Electromagnetic Wave Interactions with a Metamaterial Cloak. Phys. Rev. Lett. 99, 063903. doi:10. 1103/PhysRevLett.99.063903

Chen, H., Yang, J., Zi, J., and Chan, C. T. (2009). Transformation Media for Linear Liquid Surface Waves. Europhys. Lett. 85, 24004. doi:10.1209/0295-5075/85/ 24004

Cregan, R. F. (1999). Single-Mode Photonic Band Gap Guidance of Light in Air. Science 285, 1537-1539. doi:10.1126/science.285.5433.1537

Cui, T. J., Qi, M. Q., Wan, X., Zhao, J., and Cheng, Q. (2014). Coding Metamaterials, Digital Metamaterials and Programmable Metamaterials. Light Sci. Appl. 3, e218. doi:10.1038/lsa.2014.99

Cummer, S. A., Christensen, J., and Alù, A. (2016). Controlling Sound with Acoustic Metamaterials. Nat. Rev. Mater. 1, 16001. doi:10.1038/natrevmats. 2016.1

Cummer, S. A., and Schurig, D. (2007). One Path to Acoustic Cloaking. New J. Phys. 9, 45. doi:10.1088/1367-2630/9/3/045

Fan, C. Z., Gao, Y., and Huang, J. P. (2008). Shaped Graded Materials with an Apparent Negative Thermal Conductivity. Appl. Phys. Lett. 92, 251907. doi:10. 1063/1.2951600

Farhat, M., Enoch, S., Guenneau, S., and Movchan, A. B. (2008). Broadband Cylindrical Acoustic Cloak for Linear Surface Waves in a Fluid. Phys. Rev. Lett. 101, 134501. doi:10.1103/PhysRevLett.101.134501

Farhat, M., Guenneau, S., and Enoch, S. (2009). Ultrabroadband Elastic Cloaking in Thin Plates. Phys. Rev. Lett. 103, 024301. doi:10.1103/PhysRevLett.103.024301

Fu, Y., Shen, C., Cao, Y., Gao, L., Chen, H., Chan, C. T., et al. (2019). Reversal of Transmission and Reflection Based on Acoustic Metagratings with Integer Parity Design. Nat. Commun. 10, 2326. doi:10.1038/s41467-019-10377-9

Ge, H., Yang, M., Ma, C., Lu, M.-H., Chen, Y.-F., Fang, N., et al. (2018). Breaking the Barriers: Advances in Acoustic Functional Materials. Natl. Sci. Rev. 5, 159-182. doi:10.1093/nsr/nwx154

Genty, G., Salmela, L., Dudley, J. M., Brunner, D., Kokhanovskiy, A., Kobtsev, S., et al. (2021). Machine Learning and Applications in Ultrafast Photonics. Nat. Photon. 15, 91-101. doi:10.1038/s41566-020-00716-4

\section{AUTHOR CONTRIBUTIONS}

HC was responsible for the article in its entirety.

\section{ACKNOWLEDGMENTS}

I would like to thank my students, Ying Chen, Shan Zhu, and Pengfei Zhao for their helpful assistance.

Haldane, F. D. M., and Raghu, S. (2008). Possible Realization of Directional Optical Waveguides in Photonic Crystals with Broken Time-Reversal Symmetry. Phys. Rev. Lett. 100, 013904. doi:10.1103/PhysRevLett.100.013904

Han, T., Bai, X., Gao, D., Thong, J. T. L., Li, B., and Qiu, C.-W. (2014). Experimental Demonstration of a Bilayer Thermal Cloak. Phys. Rev. Lett. 112, 054302. doi:10.1103/PhysRevLett.112.054302

Hasan, M. Z., and Kane, C. L. (2010). Colloquium: Topological Insulators. Rev. Mod. Phys. 82, 3045-3067. doi:10.1103/RevModPhys.82.3045

Hu, G., Ou, Q., Si, G., Wu, Y., Wu, J., Dai, Z., et al. (2020). Topological Polaritons and Photonic Magic Angles in Twisted $\alpha-\mathrm{MoO}_{3}$ Bilayers. Nature 582, 209-213. doi:10.1038/s41586-020-2359-9

John, S. (1987). Strong Localization of Photons in Certain Disordered Dielectric Superlattices. Phys. Rev. Lett. 58, 2486-2489. doi:10.1103/PhysRevLett.58.2486

Khanikaev, A. B., Hossein Mousavi, S., Tse, W.-K., Kargarian, M., MacDonald, A. H., and Shvets, G. (2013). Photonic Topological Insulators. Nat. Mater. 12, 233-239. doi:10.1038/nmat3520

Kim, M., Jacob, Z., and Rho, J. (2020). Recent Advances in 2D, 3D and HigherOrder Topological Photonics. Light Sci. Appl. 9, 130. doi:10.1038/s41377-0200331-y

Knight, J. C. (2003). Photonic Crystal Fibres. Nature 424, 847-851. doi:10.1038/ nature 01940

Kushwaha, M. S., Halevi, P., Dobrzynski, L., and Djafari-Rouhani, B. (1993). Acoustic Band Structure of Periodic Elastic Composites. Phys. Rev. Lett. 71, 2022-2025. doi:10.1103/PhysRevLett.71.2022

Lai, Y., Wu, Y., Sheng, P., and Zhang, Z.-Q. (2011). Hybrid Elastic Solids. Nat. Mater. 10, 620-624. doi:10.1038/nmat3043

Leonhardt, U. (2006). Optical Conformal Mapping. Science 312, 1777-1780. doi:10.1126/science.1126493

Li, C., Xu, L., Zhu, L., Zou, S., Liu, Q. H., Wang, Z., et al. (2018). Concentrators for Water Waves. Phys. Rev. Lett. 121, 104501. doi:10.1103/PhysRevLett.121. 104501

Li, X., Lu, L., Li, J., Zhang, X., and Gao, H. (2020). Mechanical Properties and Deformation Mechanisms of Gradient Nanostructured Metals and Alloys. Nat. Rev. Mater. 5, 706-723. doi:10.1038/s41578-020-0212-2

Liu, R., Ji, C., Mock, J. J., Chin, J. Y., Cui, T. J., and Smith, D. R. (2009). Broadband Ground-Plane Cloak. Science 323, 366-369. doi:10.1126/science.1166949

Liu, Y., Leung, S., Li, F.-F., Lin, Z.-K., Tao, X., Poo, Y., et al. (2021). Bulkdisclination Correspondence in Topological Crystalline Insulators. Nature 589, 381-385. doi:10.1038/s41586-020-03125-3

Liu, Y., Liang, Z., Zhu, J., Xia, L., Mondain-Monval, O., Brunet, T., et al. (2019). Willis Metamaterial on a Structured Beam. Phys. Rev. X 9, 011040. doi:10.1103/ PhysRevX.9.011040

Liu, Z. (2000). Locally Resonant Sonic Materials. Science 289, 1734-1736. doi:10. 1126/science.289.5485.1734

Ma, G., and Sheng, P. (2016). Acoustic Metamaterials: From Local Resonances to Broad Horizons. Sci. Adv. 2, e1501595. doi:10.1126/sciadv.1501595

Ma, G., Xiao, M., and Chan, C. T. (2019). Topological Phases in Acoustic and Mechanical Systems. Nat. Rev. Phys. 1, 281-294. doi:10.1038/s42254-0190030-X

Ma, Y., Liu, Y., Raza, M., Wang, Y., and He, S. (2014). Experimental Demonstration of a Multiphysics Cloak: Manipulating Heat Flux and Electric Current Simultaneously. Phys. Rev. Lett. 113, 205501. doi:10.1103/PhysRevLett.113. 205501 
Milton, G. W., Briane, M., and Willis, J. R. (2006). On Cloaking for Elasticity and Physical Equations with a Transformation Invariant Form. New J. Phys. 8, 248. doi:10.1088/1367-2630/8/10/248

Miniaci, M., Gliozzi, A. S., Morvan, B., Krushynska, A., Bosia, F., Scalerandi, M., et al. (2017). Proof of Concept for an Ultrasensitive Technique to Detect and Localize Sources of Elastic Nonlinearity Using Phononic Crystals. Phys. Rev. Lett. 118, 214301. doi:10.1103/PhysRevLett.118.214301

Molesky, S., Lin, Z., Piggott, A. Y., Jin, W., Vucković, J., and Rodriguez, A. W. (2018). Inverse Design in Nanophotonics. Nat. Photon 12, 659-670. doi:10. 1038/s41566-018-0246-9

Ozawa, T., Price, H. M., Amo, A., Goldman, N., Hafezi, M., Lu, L., et al. (2019). Topological Photonics. Rev. Mod. Phys. 91, 015006. doi:10.1103/RevModPhys. 91.015006

Park, J. M., Cao, Y., Watanabe, K., Taniguchi, T., and Jarillo-Herrero, P. (2021). Tunable Strongly Coupled Superconductivity in Magic-Angle Twisted Trilayer Graphene. Nature 590, 249-255. doi:10.1038/s41586-021-03192-0

Park, J., Youn, J. R., and Song, Y. S. (2019). Hydrodynamic Metamaterial Cloak for Drag-free Flow. Phys. Rev. Lett. 123, 074502. doi:10.1103/PhysRevLett.123.074502

Pendry, J. B. (2006). Controlling Electromagnetic Fields. Science 312, 1780-1782. doi:10.1126/science. 1125907

Pendry, J. B., Holden, A. J., Robbins, D. J., and Stewart, W. J. (1999). Magnetism from Conductors and Enhanced Nonlinear Phenomena. IEEE Trans. Microwave Theor. Techn. 47, 2075-2084. doi:10.1109/22.798002

Pendry, J. B., Holden, A. J., Stewart, W. J., and Youngs, I. (1996). Extremely Low Frequency Plasmons in Metallic Mesostructures. Phys. Rev. Lett. 76, 4773-4776. doi:10.1103/PhysRevLett.76.4773

Pendry, J. B. (2000). Negative Refraction Makes a Perfect Lens. Phys. Rev. Lett. 85, 3966-3969. doi:10.1103/PhysRevLett.85.3966

Qian, C., Zheng, B., Shen, Y., Jing, L., Li, E., Shen, L., et al. (2020). Deep-learningenabled Self-Adaptive Microwave Cloak without Human Intervention. Nat. Photon. 14, 383-390. doi:10.1038/s41566-020-0604-2

Schittny, R., Kadic, M., Guenneau, S., and Wegener, M. (2013). Experiments on Transformation Thermodynamics: Molding the Flow of Heat. Phys. Rev. Lett. 110, 195901. doi:10.1103/PhysRevLett.110.195901

Schurig, D., Mock, J. J., Justice, B. J., Cummer, S. A., Pendry, J. B., Starr, A. F., et al. (2006). Metamaterial Electromagnetic Cloak at Microwave Frequencies. Science 314, 977-980. doi:10.1126/science.1133628

Sigalas, M., and Economou, E. N. (1993). Band Structure of Elastic Waves in Two Dimensional Systems. Solid State. Commun. 86, 141-143. doi:10.1016/00381098(93)90888-T

Silva, A., Monticone, F., Castaldi, G., Galdi, V., Alù, A., and Engheta, N. (2014). Performing Mathematical Operations with Metamaterials. Science 343, 160-163. doi:10.1126/science. 1242818

Smith, D. R. (2004). Metamaterials and Negative Refractive Index. Science 305, 788-792. doi:10.1126/science. 1096796

Smith, D. R., Padilla, W. J., Vier, D. C., Nemat-Nasser, S. C., and Schultz, S. (2000). Composite Medium with Simultaneously Negative Permeability and Permittivity. Phys. Rev. Lett. 84, 4184-4187. doi:10.1103/PhysRevLett.84.4184

Sun, S., He, Q., Xiao, S., Xu, Q., Li, X., and Zhou, L. (2012). Gradient-index MetaSurfaces as a Bridge Linking Propagating Waves and Surface Waves. Nat. Mater. 11, 426-431. doi:10.1038/nmat3292
Tang, Y., Li, L., Li, T., Xu, Y., Liu, S., Barmak, K., et al. (2020). Simulation of Hubbard Model Physics in $\mathrm{WSe}_{2} / \mathrm{WS}_{2}$ Moiré Superlattices. Nature 579, 353-358. doi:10.1038/s41586-020-2085-3

Wen, W., Zhou, L., Li, J., Ge, W., Chan, C. T., and Sheng, P. (2002). Subwavelength Photonic Band Gaps from Planar Fractals. Phys. Rev. Lett. 89, 223901. doi:10. 1103/PhysRevLett.89.223901

Willis, J. R. (1981). Variational Principles for Dynamic Problems for Inhomogeneous Elastic Media. Wave Motion 3, 1-11. doi:10.1016/01652125(81)90008-1

Xiao, M., Ma, G., Yang, Z., Sheng, P., Zhang, Z. Q., and Chan, C. T. (2015). Geometric Phase and Band Inversion in Periodic Acoustic Systems. Nat. Phys. 11, 240-244. doi:10.1038/nphys3228

Xu, H., Shi, X., Gao, F., Sun, H., and Zhang, B. (2014). Ultrathin ThreeDimensional Thermal Cloak. Phys. Rev. Lett. 112, 054301. doi:10.1103/ PhysRevLett.112.054301

Xu, Y., Fu, Y., and Chen, H. (2016). Planar Gradient Metamaterials. Nat. Rev. Mater. 1, 16067. doi:10.1038/natrevmats.2016.67

Xu, Y., Fu, Y., and Chen, H. (2015). Steering Light by a Sub-wavelength Metallic Grating from Transformation Optics. Sci. Rep. 5, 12219. doi:10.1038/ srep12219

Yablonovitch, E. (1987). Inhibited Spontaneous Emission in Solid-State Physics and Electronics. Phys. Rev. Lett. 58, 2059-2062. doi:10.1103/PhysRevLett.58. 2059

Yakovleva, A. A., Movchan, I. B., Misseroni, D., Pugno, N. M., and Movchan, A. B. (2021). Multi-Physics of Dynamic Elastic Metamaterials and Earthquake Systems. Front. Mater. 7, 620701. doi:10.3389/fmats.2020.620701

Yan, M., Lu, J., Li, F., Deng, W., Huang, X., Ma, J., et al. (2018). On-chip Valley Topological Materials for Elastic Wave Manipulation. Nat. Mater. 17, 993-998. doi:10.1038/s41563-018-0191-5

Yang, Z., Gao, F., Shi, X., Lin, X., Gao, Z., Chong, Y., et al. (2015). Topological Acoustics. Phys. Rev. Lett. 114, 114301. doi:10.1103/PhysRevLett.114. 114301

Yu, N., Genevet, P., Kats, M. A., Aieta, F., Tetienne, J.-P., Capasso, F., et al. (2011). Light Propagation with Phase Discontinuities: Generalized Laws of Reflection and Refraction. Science 334, 333-337. doi:10.1126/science.1210713

Zhou, Y., and Chen, H. (2019). Elastic Conformal Transparency. EPL 125, 54003. doi:10.1209/0295-5075/125/54003

Zou, S., Xu, Y., Zatianina, R., Li, C., Liang, X., Zhu, L., et al. (2019). Broadband Waveguide Cloak for Water Waves. Phys. Rev. Lett. 123, 074501. doi:10.1103/ PhysRevLett.123.074501

Conflict of Interest: The author declares that the research was conducted in the absence of any commercial or financial relationships that could be construed as a potential conflict of interest.

Copyright (C) 2021 Chen. This is an open-access article distributed under the terms of the Creative Commons Attribution License (CC BY). The use, distribution or reproduction in other forums is permitted, provided the original author(s) and the copyright owner(s) are credited and that the original publication in this journal is cited, in accordance with accepted academic practice. No use, distribution or reproduction is permitted which does not comply with these terms. 